University of Nebraska - Lincoln

DigitalCommons@University of Nebraska - Lincoln

October 1993

\title{
Investigation of the $\boldsymbol{\beta}-\mathbf{Y}$ phase transition in single crystal malononitrile by polarized Raman spectroscopy
}

\author{
S.J. Bonafede \\ University of Nebraska - Lincoln \\ Craig J. Eckhardt \\ University of Nebraska - Lincoln, ceckhardt1@unl.edu
}

Follow this and additional works at: https://digitalcommons.unl.edu/chemistryeckhardt

Part of the Chemistry Commons

Bonafede, S.J. and Eckhardt, Craig J., "Investigation of the $\beta$-ү phase transition in single crystal malononitrile by polarized Raman spectroscopy" (1993). Craig J. Eckhardt Publications. 17.

https://digitalcommons.unl.edu/chemistryeckhardt/17

This Article is brought to you for free and open access by the Published Research - Department of Chemistry at DigitalCommons@University of Nebraska - Lincoln. It has been accepted for inclusion in Craig J. Eckhardt Publications by an authorized administrator of DigitalCommons@University of Nebraska - Lincoln. 


\title{
Investigation of the $\beta-\gamma$ phase transition in single crystal malononitrile by polarized Raman spectroscopy
}

\author{
S. J. Bonafede and C. J. Eckhardt \\ Department of Chemistry, University of Nebraska-Lincoln, Lincoln, Nebraska 68588-0304
}

(Received 9 April 1993; accepted 9 July 1993)

\begin{abstract}
Temperature dependent, polarized Raman spectra in the lattice mode region for sublimed, single crystal malononitrile through the $\beta-\gamma$ phase transition $\left(T_{c}=294.7 \mathrm{~K}\right)$ are reported. Symmetry assignments and frequencies of vibrational modes are made and compared to previous studies. Nine out of 12 possible lattice modes are observed in each phase with three of the observed modes being affected by the phase transition. The temperature dependence of these three affected modes is attributed to cyano group and molecular dipole interactions between molecules in the crystal. The behavior of malononitrile through the $\beta-\gamma$ phase transition is analogous to the smectic $A \leftrightarrow$ nematic phase transition reported for cyano-based, liquid crystal systems.
\end{abstract}

\section{INTRODUCTION}

Malononitrile $\mathrm{CH}_{2}(\mathrm{CN})_{2}$ is reported to show remarkable solid state behavior by undergoing a unique series of displacive phase transitions. ${ }^{1}$ At temperatures above 294.7 $\mathrm{K}$, malononitrile exists in a monoclinic $\left(P 2_{1} / n\right)$ lattice. $^{2}$ Between 294.7 and $141 \mathrm{~K}$, the crystal displays triclinic $(P \overline{1}),{ }^{3}$ symmetry which reverts back to a monoclinic $\left(P 2_{1} /\right.$ $n$ ) crystal below $141 \mathrm{~K}^{4}$. While this type of transition, higher symmetry (monoclinic) $\leftrightarrow$ lower symmetry (triclinic) $\leftrightarrow$ higher symmetry (monoclinic), is thermodynamically allowed, ${ }^{5}$ malononitrile is the only known organic crystal to exhibit this phenomenon. It is more commonly seen in mixed ${ }^{6,7}$ and pure ${ }^{8,9}$ liquid crystal systems, where it is termed a "re-entrant" phase transition. A point in common for these systems and malononitrile is that they all contain cyano functional groups. This suggests that cyano groups may have a unique role in re-entrant phase transitions and the study of malononitrile may elucidate the fundamental processes causing this behavior.

The re-entrant phase transition in malononitrile was first observed by Zussman and Alexander using nuclear quadrupole resonance studies. ${ }^{1}$ A nearly contemporaneous calorimetric investigation displayed a very small specific heat anomaly between 137 and $151 \mathrm{~K}$ and virtually no anomaly at $294.7 \mathrm{~K} .{ }^{10}$ Later $\mathrm{x}$-ray diffraction results from the high $(\gamma),{ }^{2}$ intermediate $(\beta),{ }^{3}$ and low $(\alpha),{ }^{4}$ temperature forms of malononitrile indicated a slight difference of the lattice constants and crystal packing between the $\gamma$ and $\beta$ phases, while there is a larger change between the $\beta$ and $\alpha$ phases.

Variations in the crystal packing are caused by altered intermolecular forces in the lattice. Since these forces are approximately proportional to the square of the vibrational frequencies of the molecules, vibrational spectroscopy has been employed to gain insight into the modification of the intermolecular forces and to further characterize the reentrant phase transition of malononitrile.

Fujiyama and Shimanouchi have determined the 15 internal vibrational modes of the malononitrile molecule by IR, Raman, and calculative methods. ${ }^{11}$ The lattice mode region has also been investigated by Raman spectros- copy. Castellucci and Manzelli reported ten out of the 12 possible $\gamma$-phase lattice modes with symmetry assignments made from a polarized study on cut, melt-grown, single crystals of malononitrile. ${ }^{12}$ The $\gamma$-phase results of Savoie et $a{ }^{13}{ }^{13}$ from an unpolarized study on polycrystalline malononitrile showed only four out of 12 lattice modes, while the work by Bougeard et al., ${ }^{4}$ another unpolarized study on a polycrystalline $\gamma$-phase sample, reported 12 lattice modes and made symmetry assignments based on a lattice dynamics calculation.

Raman spectroscopic investigations of $\beta$-phase malononitrile were also reported by Savoie et al. ${ }^{13}$ and Bougeard et al. ${ }^{4}$ The former reported seven out of 12 possible lattice modes, while the latter located two more modes. Both studies were unpolarized and were carried out on polycrystalline samples.

The $\alpha$ phase of malononitrile was investigated by LeCalvé et al. ${ }^{14}$ using a polycrystalline sample and by Bougeard et al. ${ }^{4}$ on melt grown, cut, single crystals using Raman spectroscopy. The unpolarized study by LeCalvé et al. assigned motions to 12 lattice modes using information from isotopic frequency shifts between malononitrile and its deuterated derivative. The polarized study by Bougeard et al. produced symmetry assignments for 12 lattice modes.

Higashigaki and Wang ${ }^{15}$ followed the temperature dependence of malononitrile through the $\beta-\gamma$ phase transition by Raman spectroscopy on a melt-grown, single crystal sample. In the lattice mode region, only three modes were observed, one of which broadened above $294.7 \mathrm{~K}$ and another that split into different components below that temperature. No Raman-active optical soft mode was reported by these investigators. However, LeCalvé et al. ${ }^{14}$ followed the behavior of a soft mode throughout the $\alpha \leftrightarrow \beta \leftrightarrow \gamma$ transition sequence using Raman spectroscopy on a polycrystalline sample. An x-ray crystallographic study led Dove and Rae to postulate a motion that could correspond to a soft mode through the $\beta-\gamma$ transition. ${ }^{3}$

While numerous studies have focused on the various solid phases of malononitrile, only for the $\alpha$ phase have both symmetry and frequency assignments for all 12 Raman-active lattice modes been made. Observation and 
assignments for all lattice modes in both the $\beta$ and $\gamma$ phases remain incomplete and disagreement of assignments of currently reported modes exists among the various investigations.

Available studies do not investigate the effect of the phase transitions on all lattice modes, and there are also conflicting results regarding the occurrence of a Ramanactive optical soft mode. Among these uncertainties is that melt-grown, cut crystals, such as those used in many of the previous studies, are expected to have quite high defect concentrations and substantial residual internal strain, ${ }^{16}$ both of which may affect the bandwidths and frequencies of lattice modes.

Many problems remain concerning the phase transitions occurring in malononitrile: (1) incomplete lattice mode assignments for the $\beta$ and $\gamma$ phases; (2) lack of temperature dependent information on several of the lattice modes through the $\beta-\gamma$ phase transition: (3) discrepancies concerning the occurrence of a Raman-active soft mode; and (4) the effect of melt-grown, cut crystals or polycrystalline samples on the lattice modes and their spectra. To address these problems, a temperature dependent, polarized, Raman spectroscopic study of the low frequency $\left(<200 \mathrm{~cm}^{-1}\right)$ vibrations of the $\beta$ and $\gamma$ phases of sublimed, uncut, single crystal malononitrile was undertaken.

Section II of this paper describes the purification, growth, and preliminary characterization of the malononitrile crystal and the instrumentation used for data collection. The results from the polarized Raman study of both $\beta$ - and $\gamma$-phase malononitrile are presented in Sec. III. Frequency and symmetry assignments of vibrational modes made directly from the observed spectra in both phases are subsequently presented and discussed. A comparison between results obtained here and previous work, together with a discussion of the $\beta-\gamma$ phase transition occurring in malononitrile, is given in Sec. IV.

\section{EXPERIMENT}

Malononitrile was obtained from Aldrich Chemical Co. The original yellowish-brown material was purified by repeated vacuum sublimations. The resulting material appeared as a white powder. Clear, well-developed, single crystals of the $\gamma$ phase were grown by sublimation at 40 $m$ Torr and a temperature of $298 \mathrm{~K}$. The crystal exhibits monoclinic $\left(P 2_{1} / n\right)$ symmetry in the $\gamma$ phase with four molecules in the primitive unit cell, ${ }^{2}$ while the $\beta$ phase is triclinic $(P \overline{1})$ and also with four molecules in the primitive unit cell. ${ }^{3}$ The cell parameters for both phases are given in Table I. Crystal face assignments were made by optical goniometry. The Euler angles for both phases which describe the orientation of the molecular axes with respect to the unit cell axes are also given in Table I. It is seen that the unit cell $b$ axis coincides with the molecular $y$ axis. The crystallographic convention of setting the $b$ axis as the unique axis for monoclinic systems will be followed throughout this paper.

Since both the propagation and polarization directions of light may deviate inside an anisotropic dielectric medium, ${ }^{17}$ the effect of crystal optics must be considered. This
TABLE I. Lattice parameters for malononitrile $\gamma$ and $\beta$ phases. $\phi, \psi$, and $\theta$ are the Euler angles.

\begin{tabular}{ccc}
\hline \hline Lattice parameters & $\gamma$ phase $\left(P_{1} / n\right)$ & $\beta$ phase $(P \overline{1})$ \\
\hline$a(\AA)$ & 7.84 & 7.82 \\
$b(\AA)$ & 7.63 & 7.63 \\
$c(\AA)$ & 6.18 & 6.15 \\
$\alpha$ (degrees) & 90.00 & 89.68 \\
$\beta$ (degrees) & 96.20 & 96.80 \\
$\gamma$ (degrees) & 90.00 & 90.23 \\
$Z$ & 4 & 4 \\
$\phi$ (degrees) & 14.01 & 23.16 \\
$\theta$ (degrees) & 0.071 & 3.308 \\
$\psi$ (degrees) & 16.10 & 16.46 \\
\hline \hline
\end{tabular}

correction has not been accounted for in other studies of malononitrile. The refractive indices at $275 \mathrm{~K}$ along the $a$, $b$, and $c^{*}$ axes at $5145 \AA$ were determined by polarized, specular reflection spectroscopy. ${ }^{18}$ The optical indicatrix at $5145 \AA$ that was obtained from these measurements was used to calculate the changes of the propagation and polarization directions of light inside the crystal. The polarization directions for the $\beta$ phase are found $4^{\circ}$ counterclockwise relative to the $a$ - and $c^{*}$-crystallographic axes for $\beta$-phase malononitrile. This slight change of polarization directions shows the optical indicatrix remains essentially constant at $5145 \AA$ on the transition from the triclinic to the monoclinic phase. This is consistent with the report of Castellucci et al. that the polarization patterns of infrared spectra for the $\beta$ and $\gamma$ phases do not change, thereby indicating that the monoclinic axes do not tilt noticeably through the phase transition. ${ }^{19}$ Because the optical indicatrix varies only slightly through the phase transition and the crystal structures of the $\beta$ and $\gamma$ phases differ only slightly, the triclinic $\beta$ phase can be treated as pseudomonoclinic. For ease of conccptualization and reference, all subsequent results will be analyzed with monoclinic pseudosymmetry for the $\beta$ phase.

The symmetry representations for malononitrile's 21 optical lattice vibrations for the monoclinic lattices are

$$
\Gamma_{\beta, \gamma}=6 A_{g}+6 B_{g}+5 A_{u}+4 B_{u} .
$$

For both the monoclinic $\gamma$ phase and pseudomonoclinic $\beta$ phase, the $A_{g}$ Raman modes are active for the polarization (Porto notation) $)^{20}$ configurations $(a a),(b b),\left(c^{*} c^{*}\right)$, and $\left(a c^{*}\right)$, while the $B_{g}$ modes are active for the $(a b)$ and ( $\left.b c^{*}\right)$ configurations.

The Raman spectrometer used for data acquisition was based on a design by Tobin ${ }^{21}$ with the optical and electronic components described by White. ${ }^{22}$ A SPEX 1400 double monochromator with $1800 \mathrm{~g} / \mathrm{mm}$ holographic gratings used as the dispersing elements was calibrated with known lines from a high pressure mercury lamp. The tempcratures for subambient studies were achieved using a cooled $\mathrm{N}_{2}$ gas flow apparatus. ${ }^{23}$ The temperature was monitored by a platinum sensor and controlled with a $\mathrm{Ni}$ chrome heater wire used in conjunction with a Lake Shore Cryotronics Inc. DRC-84C temperature controller. The temperatures used in the Raman experiments were accu- 
TABLE II. Experimental mode frequencies and symmetries of malononitrile for the six polarization configurations. $\beta$-phase values are in parentheses.

\begin{tabular}{|c|c|c|c|c|c|}
\hline $\begin{array}{c}(a a) \\
A_{g}\end{array}$ & $\begin{array}{c}\text { Polari } \\
(b b) \\
A_{g}\end{array}$ & $\begin{array}{c}\left(c^{*} c^{*}\right) \\
A_{g}\end{array}$ & $\begin{array}{c}\text { ations and } \\
\qquad A_{g}\end{array}$ & $\begin{array}{c}\text { mmetries } \\
(a b) \\
B_{g}\end{array}$ & $\begin{array}{c}\left(b c^{*}\right) \\
B_{g}\end{array}$ \\
\hline $\begin{array}{c}49 \mathrm{~m} \\
(49 \mathrm{~m})\end{array}$ & $\begin{array}{c}49 \mathrm{~m} \\
(49 \mathrm{~m})\end{array}$ & & & & \\
\hline $\begin{array}{c}53 w \\
(57 w)\end{array}$ & $\begin{array}{c}53 w \\
(53 w)\end{array}$ & $\begin{array}{c}? \\
(57)\end{array}$ & $\begin{array}{c}53 \mathrm{~s} \\
(57 \mathrm{~s})\end{array}$ & & $\begin{array}{c}? \\
(57 \mathrm{br})\end{array}$ \\
\hline & & $\begin{array}{c}? \\
(74)\end{array}$ & $\begin{array}{l}74 \mathrm{~s} \\
(74 \mathrm{~s})\end{array}$ & & \\
\hline \multirow[t]{4}{*}{$\begin{array}{c}84 w \\
(87 w)\end{array}$} & $\begin{array}{c}84 w \\
(87 w)\end{array}$ & $\begin{array}{c}? \\
(87)\end{array}$ & $\begin{array}{c}84 \mathrm{sh} \\
(87 \mathrm{sh})\end{array}$ & & \\
\hline & & & & $\begin{array}{c}87 \mathrm{~s} \\
(87 \mathrm{~s})\end{array}$ & \\
\hline & & & & & $\begin{array}{c}91 \mathrm{~s} \\
(91 \mathrm{~s}) \\
108 \mathrm{sh} \\
(111 \mathrm{sh})\end{array}$ \\
\hline & & $\stackrel{?}{(117)}$ & $\begin{array}{c}117 \mathrm{~h} \\
(117 \mathrm{~h})\end{array}$ & & \\
\hline \multirow[t]{2}{*}{$\begin{array}{c}156 \mathrm{~s} \\
(156 \mathrm{~s})\end{array}$} & $\begin{array}{c}156 \mathrm{~s} \\
(156 \mathrm{~s})\end{array}$ & & & $\begin{array}{c}? \\
(156 \mathrm{br})\end{array}$ & $\begin{array}{c}? \\
(156 \mathrm{br})\end{array}$ \\
\hline & & & & $\begin{array}{c}181 \mathrm{~m} \\
(181 \mathrm{~m})\end{array}$ & $\begin{array}{c}181 \mathrm{~m} \\
(181 \mathrm{~m})\end{array}$ \\
\hline
\end{tabular}

rate to $\pm 0.5 \mathrm{~K}$. A Coherent Innova $90 \mathrm{Ar}^{+}$laser operating at $5145 \AA$ was the excitation source with a power of 50 $\mathrm{mW}$ at the sample. Spectra were digitally recorded in 1 $\mathrm{cm}^{-1}$ intervals from 4 to $200 \mathrm{~cm}^{-1}$.

\section{RESULTS}

Temperature dependent Raman spectra of single crystal malononitrile from 30 to $200 \mathrm{~cm}^{-1}$ taken in the $(a b)$, $(a a),(b b),\left(a c^{*}\right),\left(b c^{*}\right)$, and $\left(c^{*} c^{*}\right)$ polarization configurations are shown in Figs. 1-6. The frequencies and symmetry assignments of the Raman-active, vibrational modes of $\beta$ - and $\gamma$-phase malononitrile seen in these spectra are given in Table II. Despite the various assumptions used, a method employing a curve-fitting routine that models Raman modes with a Lorentzian band profile was used to assign frequencies to vibrations that appeared in spectral envelopes comprised of more than one peak. Weak vibrational peaks in scattering configurations which have intense peaks at the same frequency in different configurations were assigned as breakthrough scattering. These modes occur from depolarization of the incident and scattered light due to either surface roughness or internal defects. ${ }^{22}$

The spectral region from 4 to $30 \mathrm{~cm}^{-1}$ was studied for the $\beta$ phase at $T=275 \mathrm{~K}$. No Raman-active vibrations were observed in this region. Attempts to investigate this region in the $\gamma$ phase were not successful due to increased Rayleigh broadening.

The low-frequency Raman spectra of $B_{g}$ symmetry in the $(a b)$ configuration for both phases are shown in Fig. 1. A very intense peak is observed at $87 \mathrm{~cm}^{-1}$, while a medium strength peak is seen at $181 \mathrm{~cm}^{-1}$. Also, in the $\beta$ phase below $292 \mathrm{~K}$, a very weak peak attributed to break-

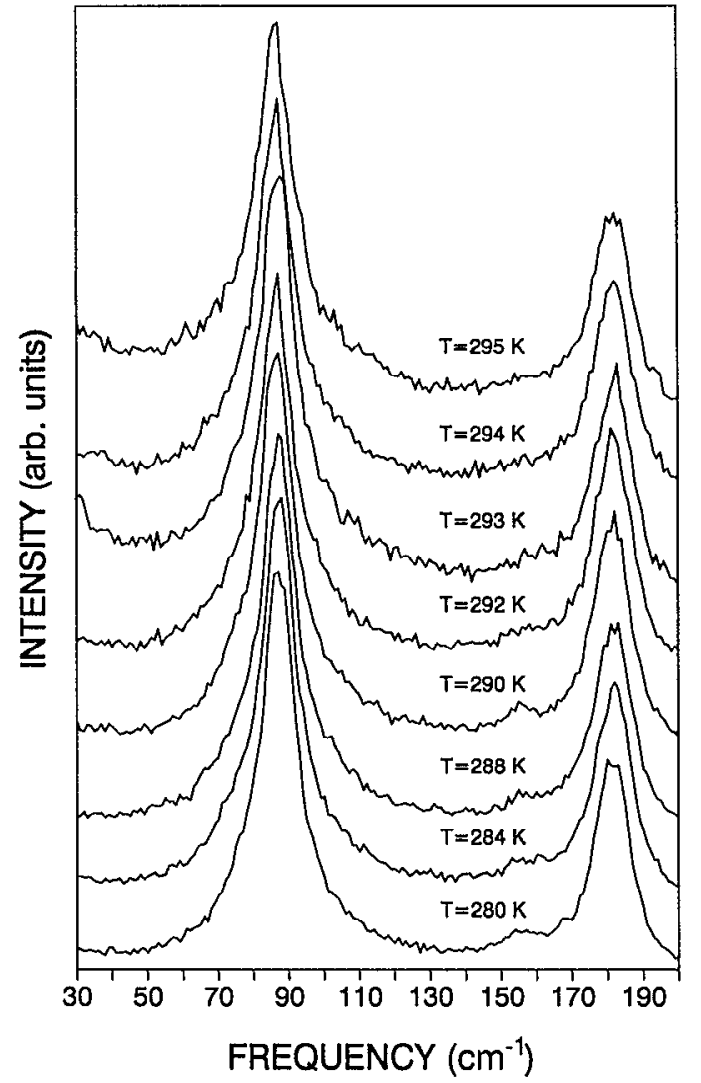

FIG. 1. Polarized, $B_{g}$-active, Raman spectra in the $(a b)$ scattering configuration.

through scattering is observed at $156 \mathrm{~cm}^{-1}$. The spectra for both phases show no evidence of temperature dependence of any of the observed bands, although broadening of the Rayleigh wing is observed at temperatures higher than $293 \mathrm{~K}$.

Figure 2 shows the $\left(a c^{*}\right)$ series of spectra which display $\boldsymbol{A}_{g}$ symmetry modes in both the $\beta$ and $\gamma$ phases. Strong peaks at 57 and $74 \mathrm{~cm}^{-1}$, a shoulder at $87 \mathrm{~cm}^{-1}$, and a very broad structure between $115-120 \mathrm{~cm}^{-1}$ are observed in the $\beta$ phase. The $\gamma$ phase has strong peaks at 53 and $74 \mathrm{~cm}^{-1}$, a shoulder at $84 \mathrm{~cm}^{-1}$, and also exhibits a broad structure from 115 to $120 \mathrm{~cm}^{-1}$. The band found in both phases at $156 \mathrm{~cm}^{-1}$ is attributed to breakthrough. The Rayleigh peak broadens above $294 \mathrm{~K}$.

On passing through the transition point, the $\beta$ phase 57 $\mathrm{cm}^{-1}$ mode shifts to $53 \mathrm{~cm}^{-1}$ in the $\gamma$ phase and also broadens. The peak at $74 \mathrm{~cm}^{-1}$ remains constant through the transition, but the shoulder at $87 \mathrm{~cm}^{-1}$, which is resolved in the $\beta$ phase, disappears in the $\gamma$ phase. The best fit from the Lorentzian curve-fitting routine indicates the $\beta$ phase $87 \mathrm{~cm}^{-1}$ mode shifts to $84 \mathrm{~cm}^{-1}$ in the $\gamma$ phase. This results in a coalescing of the $74 \mathrm{~cm}^{-1}$ and the high energy shoulder in the $\gamma$ phase and leads to the observed $\left(a c^{*}\right)$ spectral profile.

The $(a a)$ and $(b b)$ scattering configurations (Figs. 3 and 4) for $\beta$-phase spectra show medium intensity peaks representing $A_{g}$ active modes at 49,57 , and $85 \mathrm{~cm}^{-1}$, and a very intense peak corresponding to a mode at $156 \mathrm{~cm}^{-1}$, 


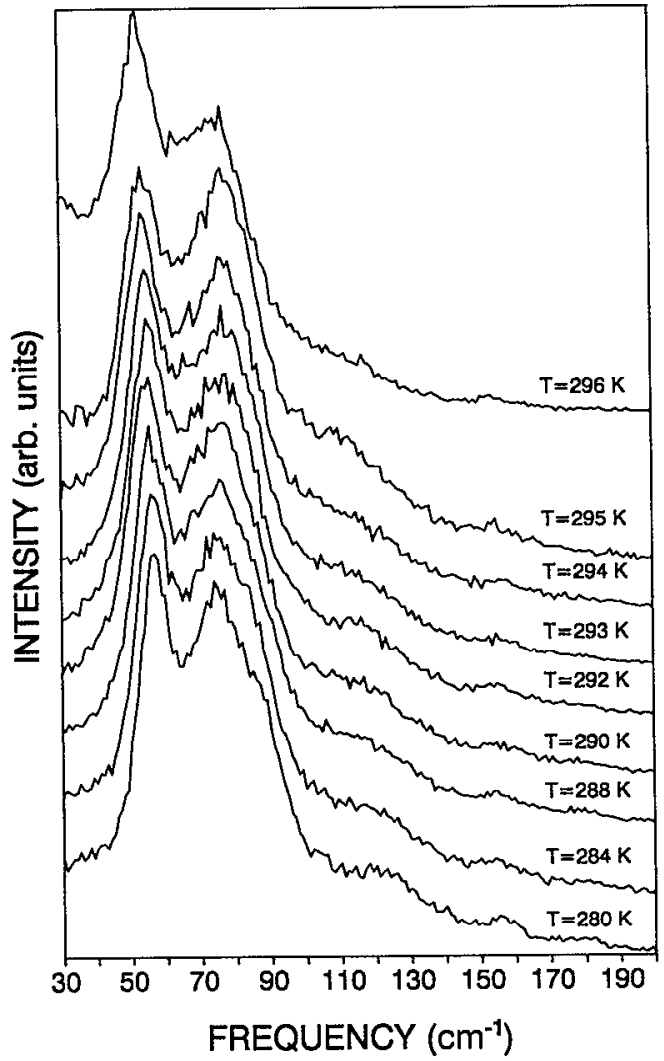

FIG. 2. Polarized, $A_{g}$-active, Raman spectra in the ( $\left.a a\right)$ scattering configuration.

while the $\gamma$-phase $A_{g}$ active modes are seen at $49,53,85$, and $156 \mathrm{~cm}^{-1}$. The breakthrough structure at $156 \mathrm{~cm}^{-1}$ seen in the $(a b)$ and $\left(a c^{*}\right)$ configurations is attributed to the $156 \mathrm{~cm}^{-1}$ mode seen in these spectra. As the temperature of the $\beta$ phase rises towards the transition temperature, the peak at $57 \mathrm{~cm}^{-1}$ shifts to $53 \mathrm{~cm}^{-1}$ and coalesces with the $49 \mathrm{~cm}^{-1}$ peak at $294 \mathrm{~K}$ in a similar fashion to that reported for the $\left(a c^{*}\right)$ configuration.

Figure 5 displays the $\left(b c^{*}\right)$ series of spectra. The $\beta$-phase $B_{g}$ vibrations include an intense peak at $91 \mathrm{~cm}^{-1}$ with a pronounced shoulder at $111 \mathrm{~cm}^{-1}$, a medium strength peak at $181 \mathrm{~cm}^{-1}$ and breakthrough structure at 57 and $156 \mathrm{~cm}^{-1}$ from the 57 and the $156 \mathrm{~cm}^{-1}$ modes seen in the $\left(a c^{*}\right)$ and $(b b)$ configurations. The $B_{g}$ symmetry modes of the $\gamma$ phase result in an intense peak at 91 $\mathrm{cm}^{-1}$ with a shoulder at $108 \mathrm{~cm}^{-1}$, and a medium intensity peak at $181 \mathrm{~cm}^{-1}$. Breakthrough attributed to the 156 $\mathrm{cm}^{-1}$ mode in the $(b b)$ configuration also occurs at 156 $\mathrm{cm}^{-1}$ in these spectra. Extensive Rayleigh broadening is observed above $294 \mathrm{~K}$.

The $\beta$-phase envelope which contains the $91 \mathrm{~cm}^{-1}$ peak and the prominent shoulder at $111 \mathrm{~cm}^{-1}$ coalesces into a broad, unresolved band on transformation to the $\gamma$ phase. Three possibilities exist which can cause this spectral behavior: (1) the $91 \mathrm{~cm}^{-1}$ peak shifts to higher energy, while the $111 \mathrm{~cm}^{-1}$ peak remains constant; (2) the 91 $\mathrm{cm}^{-1}$ peak stays constant, while the $111 \mathrm{~cm}^{-1}$ peak shifts to lower energy; and (3) the $91 \mathrm{~cm}^{-1}$ peak shifts to higher

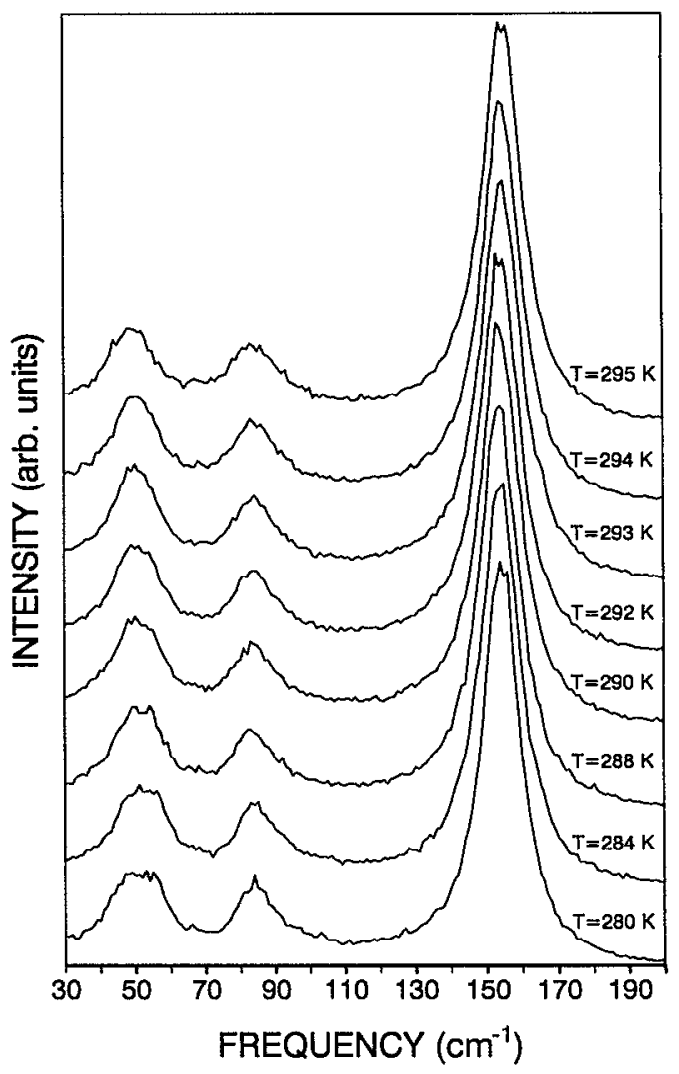

FIG. 3. Polarized, $A_{g}$-active, Raman spectra in the $(b b)$ scattering configuration.

energy, while the $111 \mathrm{~cm}^{-1}$ peak shifts to lower energy. The results from the curve-fitting routine coupled with visual inspection indicated that the change in the spectral envelope was best described by the second possibility.

Figure 6 shows the $\left(c^{*} c^{*}\right)$ series of spectra. Attempts on several crystals to follow the temperature dependence of the $A_{g}$-active modes into the $\gamma$ phase were unsuccessful due to extreme Rayleigh broadening close to $294 \mathrm{~K}$. The low signal-to-noise ratio that is seen in this scattering configuration is attributed to low scattering intensity. This is also seen in previous work. ${ }^{12}$ However, vibrational modes in the $\beta$ phase are still observed at $57,74,87$, and $117 \mathrm{~cm}^{-1}$.

\section{DISCUSSION}

\section{A. Comparison to previous results}

Below $200 \mathrm{~cm}^{-1}$, only nine lattice modes are seen for both the $\beta$ and $\gamma$ phases. In the pseudomonoclinic $\beta$ phase and in the monoclinic $\gamma$ phase, all six $A_{g}$ active, but only three of the six $B_{g}$-active lattice modes are assigned. The vibrational mode at $181 \mathrm{~cm}^{-1}$ in both the $\beta$ and $\gamma$ phases is assigned as the cyano-C-cyano intramolecular bending mode of the malononitrile molecule in agreement with Castellucci and Manzelli ${ }^{12}$ but contrary to three other previous studies. Bougeard el al. ${ }^{4}$ and LeCalvé et al. ${ }^{14}$ suggest that the modes at 156 and $181 \mathrm{~cm}^{-1}$ are internal modes resulting from correlation splitting, while Higashigaki and 


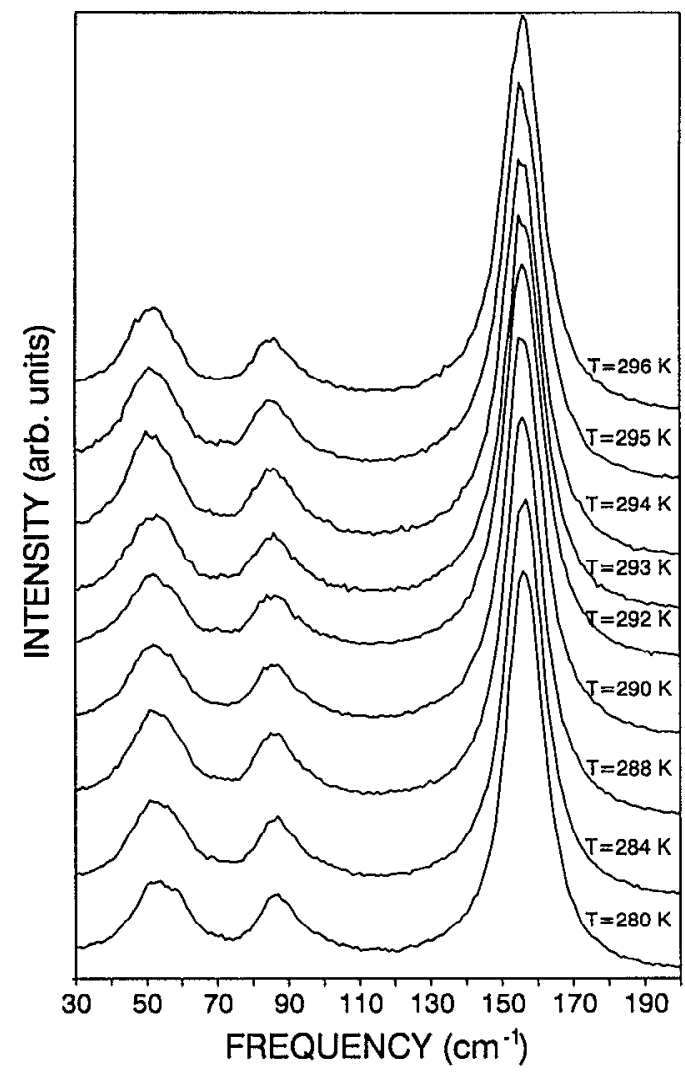

FIG. 4. Polarized, $A_{g}$-active, Raman spectra in the ( $\left.a c^{*}\right)$ scattering configuration.

Wang ${ }^{15}$ assign the mode at $181 \mathrm{~cm}^{-1}$ as a lattice vibration, but assign the $156 \mathrm{~cm}^{-1}$ mode as the cyano-C-cyano internal mode.

To support the assignment of a lattice mode at 156 $\mathrm{cm}^{-1}$ and the cyano-C-cyano internal bending mode at $181 \mathrm{~cm}^{-1}$, the cyano-C-cyano bend and the $\mathrm{X}-\mathrm{C}-\mathrm{X}$ bending vibration of a series of disubstituted methancs are compared, where $\mathrm{X}$ represents halogen atoms $\mathrm{Cl}, \mathrm{Br}$, and $\mathrm{I}$.

A stereographic projection of $\gamma$ - and $\beta$-phase malononitrile $2^{\circ}$ from the normal of the $b c$ plane is shown in Fig. 7. Unshaded molecules are those whose $\mathrm{C}-\mathrm{C}-\mathrm{C}$ angle apices are in the $-b$ direction, while mottled molecules have their $\mathrm{C}-\mathrm{C}-\mathrm{C}$ angle apices in the $+b$ direction. Distinct layers of molecules are oriented parallel to the $b$ axis. This same layering motif occurs in both $\mathrm{CH}_{2} \mathrm{Br}_{2}$ and $\mathrm{CH}_{2} \mathrm{I}_{2} \cdot{ }^{24}$ $\mathrm{CH}_{2} \mathrm{Cl}_{2}$ also exhibits layering, but with molecules in altcrnating layers being rotated with respect to each other. ${ }^{25}$

Along with the similar crystal packing, both the $\mathrm{CH}_{2} \mathrm{X}_{2}$ molecules and malononitrile have tetrahedral molecular structure, and there are numerous analogies of the chemistry of the cyano group and halogens. ${ }^{26}$ From these, one would expect that the $\mathrm{CH}_{2} \mathrm{X}_{2}$ molecules and crystals are reasonable model systems for malononitrile, and the vibrations occurring in these systems can aid in the assignment of the low frequency vibrations in malononitrile.

Upon crystallization from the liquid state, the low frequency Raman $\mathrm{X}-\mathrm{C}-\mathrm{X}$ bending modes shift from 121 to $132 \mathrm{~cm}^{-1}$ for $\mathrm{CH}_{2} \mathrm{I}_{2}$ at $195 \mathrm{~K}$, from 175 to $179 \mathrm{~cm}^{-1}$ for

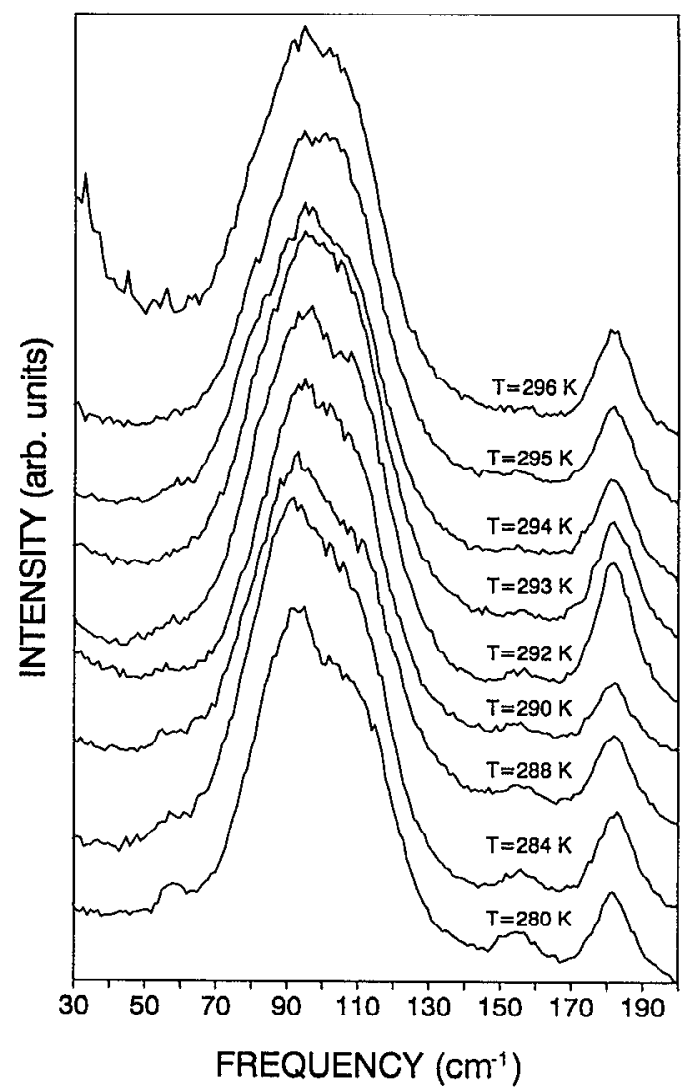

FIG. 5. Polarized, $B_{z}$-active, Raman spectra in the $\left(b c^{*}\right)$ scattering configuration.

$\mathrm{CH}_{2} \mathrm{Br}_{2}$ at $195 \mathrm{~K}$, and from $284 \mathrm{~cm}^{-1}$ to form a doublet at 285 and $291 \mathrm{~cm}^{-1}$ for $\mathrm{CH}_{2} \mathrm{Cl}_{2}$ at $77 \mathrm{~K}^{27}$ The highest reported frequencies for lattice vibrations are at $89 \mathrm{~cm}^{-1}$ for $\mathrm{CH}_{2} \mathrm{I}_{2} 96 \mathrm{~cm}^{-1}$ for $\mathrm{CH}_{2} \mathrm{Br}_{2}$, and $113 \mathrm{~cm}^{-1}$ for $\mathrm{CH}_{2} \mathrm{Cl}_{2}$. Only $\mathrm{CH}_{2} \mathrm{Cl}_{2}$ shows correlation splitting of the internal bending vibrations where both solid state components occur at higher frequencies than in the liquid state.

The frequency increase upon crystallization for the $\mathrm{X}-\mathrm{C}-\mathrm{X}$ bending mode of the dihalogen methane molecules also appears to occur for the cyano-C-cyano internal bending mode in malononitrile, where it appears at 167 $\mathrm{cm}^{-1}$ in the neat liquid. ${ }^{11}$ The results reported here for crystalline malononitrile show that the closest frequencies to the liquid cyano-C-cyano bending frequency occur at 156 and $181 \mathrm{~cm}^{-1}$. Although correlation splitting, which, in analogy with $\mathrm{CH}_{2} \mathrm{Cl}_{2}$ would have components at higher frequency than the liquid state bending frequency, does not appear to occur in malononitrile, it would, if present, cause the frequency to be shifted to higher energy than in the liquid. Also, following the behavior of the $\mathrm{X}-\mathrm{C}-\mathrm{X}$ bending vibration in the dihalomethanes, the cyano-C-cyano bending vibration should shift to higher frequency on going from the liquid to the solid state. From these considerations, the $181 \mathrm{~cm}^{-1}$ mode is attributed to the cyano-Ccyano bending mode of malononitrile, while that at 156 $\mathrm{cm}^{-1}$ is assigned as a lattice mode.

The $\gamma$-phase spectra permit identification of all six $A_{g^{-}}$ active modes, although only three $B_{g}$-active modes are 


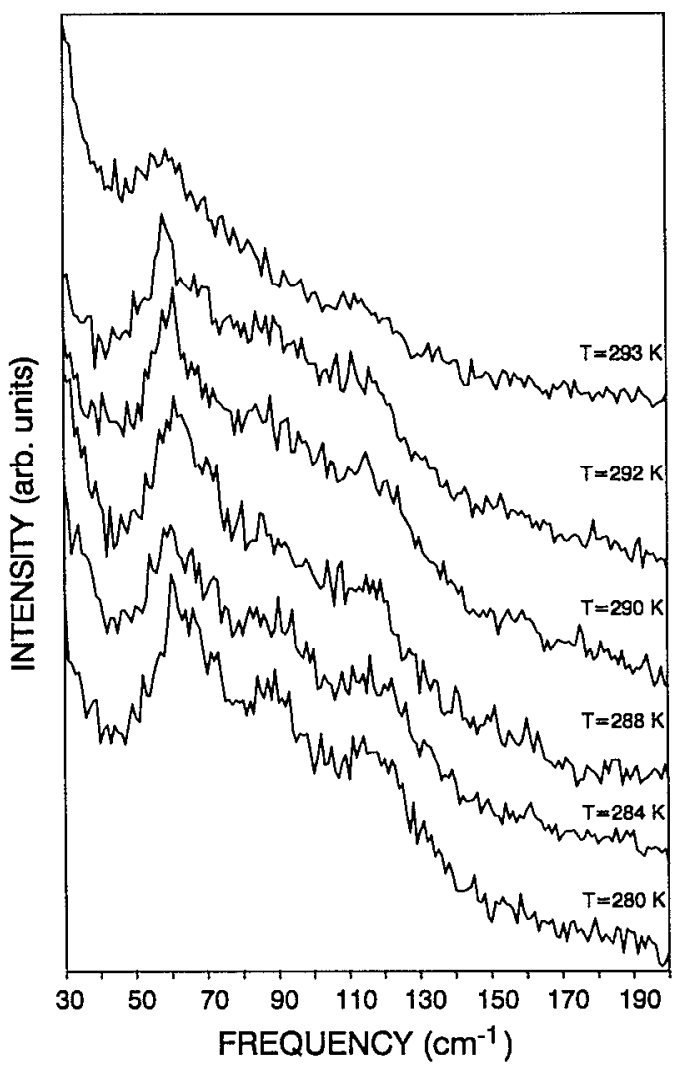

FIG. 6. Polarized, $A_{g}$-active, Raman spectra in the $\left(c^{*} c^{*}\right)$ scattering configuration.

found. The $A_{g}$-active bands at 74,84 , and $156 \mathrm{~cm}^{-1}$ and the $B_{g}$-active modes at 87 and $108 \mathrm{~cm}^{-1}$ observed in our spectra were reported by Castellucei and Manzelli, ${ }^{12}$ but the $A_{g}$-active modes observed here at 49 and $53 \mathrm{~cm}^{-1}$ were reported at 52 and $55 \mathrm{~cm}^{-1}$ in the earlier work. These discrepancies are attributed to the earlier workers' use of melt-grown, cut, single crystals. The $A_{p}$-active mode that leads to the broad structure at $117 \mathrm{~cm}^{-1}$ and the $B_{g}$-active mode at $91 \mathrm{~cm}^{-1}$ observed in this study were not reported in the earlier work, although a previously reported $A_{g}$ active mode at $80 \mathrm{~cm}^{-1}$ for the $\left(a c^{*}\right)$ configuration was not reproduced in our measurements.

The $\gamma$-phase $A_{g}$-active modes reported by Bougeard et $a l^{4}$ at 49 and $115 \mathrm{~cm}^{-1}$ agree with vibrations observed here, while modes they reported at 55,78 , and $88 \mathrm{~cm}^{-1}$ are at higher frequencies than our observations at 53,74 , and $84 \mathrm{~cm}^{-1}$. A $B_{\mathrm{g}}$-active mode reported at $88 \mathrm{~cm}^{-1}$ corresponds to our results, but a mode at $104 \mathrm{~cm}^{-1}$ is lower in frequency than found at $108 \mathrm{~cm}^{-1}$. These differences are attributed to difficulties with strain and breakthrough scattering as well as uncertainties of measurements on polycrystalline samples in comparison to measurements on single crystals. Bougeard et al. reported a $B_{\mathrm{g}}$-active mode at 7 $\mathrm{cm}^{-1}$ and an $A_{g}$-active mode at $68 \mathrm{~cm}^{-1}$ which could not be reproduced, while the $B_{g}$-active $91 \mathrm{~cm}^{-1}$ mode, the $A_{g}$-active $156 \mathrm{~cm}^{-1}$ lattice mode, and the $181 \mathrm{~cm}^{-1}$ internal bending mode observed here were not reported in the previous study.
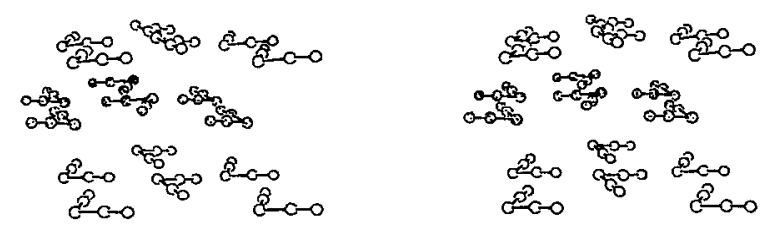

a
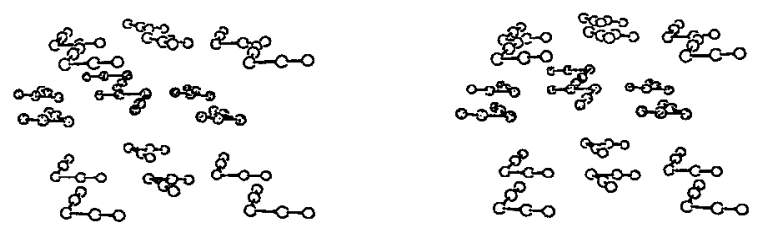

b

FIG. 7. A stereographic view of $\gamma$ - and $\beta$-phase malononitrile molecules $2^{\circ}$ from the normal of the $b c$ plane. Unshaded molecules have their $\mathrm{C}-\mathrm{C}-\mathrm{C}$ angle apices directed along the $-b$ axis, while motled molecules are directed along the $+b$ axis.

Beside these differences, Bougeard et al. report more than one mode occurring at the same frequency. They suggested that three vibrations have a frequency of $88 \mathrm{~cm}^{-1}$ with two of the modes being of $B_{g}$ symmetry and the other of $A_{g}$ symmetry. Also, two modes, one each of $A_{g}$ and $B_{g}$ symmetry, were assigned at $78 \mathrm{~cm}^{-1}$ and an $A_{g}$ and a $B_{g}$ mode were both assigned at $115 \mathrm{~cm}^{-1}$. These experimentally determined modes were all fit to results from a lattice dynamics calculation.

While it is possible that vibrations of different symmetry may be accidentally degenerate or near degenerate, they would be difficult to resolve in an unpolarized study on a polycrystalline sample as was previously reported. However, accidentally degenerate modes of the same symmetry such as the two $B_{g}$-active modes reported at 88 $\mathrm{cm}^{-1}$ are expected to interact and split. This was not considered in the previous study. While frequency proximity criteria of experimental vibrational frequencies and symmetries to lattice dynamics calculations can be helpful for experimental mode assignments, those reported by Bougeard et al. do not appear to be well-substantiated in this case.

The unpolarized study of Bougeard et al. ${ }^{4}$ on $\beta$-phase malononitrile reported nine modes. Modes reported at 74 and $158 \mathrm{~cm}^{-1}$ correspond to modes observed in our study, while modes at 53 and $83 \mathrm{~cm}^{-1}$ were seen in our spectra at 57 and $87 \mathrm{~cm}^{-1}$. Bougeard et al. also reported modes at 14 , 62,100 , and $135 \mathrm{~cm}^{-1}$ which have no corresponding structure in our spectra. Differences are again attributed to sample quality and preparation.

Two of the $\beta$-phase modes from Bougeard $e t$ al. at 92 and $110 \mathrm{~cm}^{-1}$ were correlated to $A_{g}$ symmetry modes occurring in the $\gamma$ phase. Our results show $\beta$-phase modes at 
91 and $111 \mathrm{~cm}^{-1}$ in the $\left(b c^{*}\right)$ configuration which are of $B_{g}$ symmetry. The greater information content of the polarized Raman measurements reported here afford a more reliable assignment than can be obtained solely from frequency proximities.

Special attention has been given to the frequency region below $30 \mathrm{~cm}^{-1}$. A Raman-active optical soft mode was reported by LeCalvé et al. ${ }^{14}$ while results reported by Higashigaki and Wang ${ }^{15}$ show no evidence of this mode. Our investigation also does not support the assignment of a Raman active soft mode below $30 \mathrm{~cm}^{-1}$. However, it is possible that such a mode exists, but is obscured by the Rayleigh wing.

The investigation by Higashigaki and Wang ${ }^{15}$ of the temperature dependence of lattice modes in the $(a a)$ and (ab) scattering configurations through the $\beta-\gamma$ phase transition showed broadening of a mode at $90 \mathrm{~cm}^{-1}$ in the $(a b)$ configuration and, in the $(a a)$ configuration, displayed splitting of a mode at $90 \mathrm{~cm}^{-1}$ below $294.7 \mathrm{~K}$, while all other modes remained unaffected. The $(a b)$ scattering spectra shown in Fig. 4 have the same structure as reported by Higashigaki and Wang, but with two differences-the mode reported at $90 \mathrm{~cm}^{-1}$ in their study is observed here at $87 \mathrm{~cm}^{-1}$ with no appreciable broadening and the relative band shapes of the $(a a)$ spectra in Fig. 5 differ. Their spectra exhibit a greater number of modes and strikingly different peak height ratios. Also, modes observed in this work that shifted from 57 to $53 \mathrm{~cm}^{-1}$ and from 87 to 84 $\mathrm{cm}^{-1}$ for the $\left(a c^{*}\right)$ configuration, and from 111 to 108 $\mathrm{cm}^{-1}$ for the $\left(b c^{*}\right)$ configuration were not reported by Higashigaki and Wang.

The differences in mode frequencies and the broadening of the $90 \mathrm{~cm}^{-1}$ mode of the Higashigaki and Wang $(a b)$ spectra with respect to our results may be attributed to sample quality arising from the different sample growth procedures. Furthermore, their experiments were performed with a sample in a glass ampoule making the polarization directions of the incident and scattered light difficult to orient along the crystallographic axes of the crystal. The ampoule itself could have introduced depolarization. Consequently, $(a a)$ spectra could have been obtained which appear to have a component of $(a b)$ spectral character. The breakthrough of the $(a b)$ spectra would lead to mixed $(a a)$ spectra which generate the apparent mode splitting and the peak height ratio variances reported by Higashigaki and Wang.

\section{B. Analysis of $\beta-\gamma$ phase transition}

The $\beta-\gamma$ phase transition is reflected by the behavior of several of the lattice vibrations of malononitrile. Since the relative orientations of the malononitrile molecules do not show large deviations from the unit cell axes (see the Euler angles in Table I), the polarization configurations provide information about the most prevalent motions of the molecules during the unit cell vibrations. The $A_{g}$-active vibrations obtained in the $\left(a c^{*}\right)$ configuration in the pseudomonoclinic $\beta$ and monoclinic $\gamma$ phases are predominantly librations in the $a c$ plane ( $b$ axis), while the $B_{g^{-}}$ active vibrations of both phases obtained in the $\left(b c^{*}\right)$ con- figuration are predominantly $b c$-plane ( $a$-axis) librations. From the Raman data, these motions can be determined and then analyzed to see what molecular interactions may cause the frequency shifting of the affected modes.

Dye and Eckhardt ${ }^{28}$ and White and Eckhardt ${ }^{29}$ have shown that functional group interaction of a halogenated hydrocarbon manifests itself during a structural phase transition. Thus, it is important to investigate the cyano group interactions to determine what effects they have during the $\beta-\gamma$ phase transition. The distance between the geometric center points of the closest cyano groups of molecules in which the apices of the $\mathrm{C}-\mathrm{C}-\mathrm{C}$ angle point in the same direction is $3.86 \AA$ in the $\beta$ phase and $3.84 \AA$ in the $\gamma$ phase. These molecules are designated as syn molecules. For molecules in which the apices of the $\mathrm{C}-\mathrm{C}-\mathrm{C}$ angle point in opposing directions, or anti molecules, this distance in the $\beta$ phase is $3.80 \AA$ and in the $\gamma$ phase is $3.77 \AA$. One-half of the effective thickness of aromatic hydrocarbons $1.7 \AA$ (Ref. 30) was used to approximate the van der Waals interaction distance for the cyano groups. The distance between cyano groups for both syn- and anti molecules is significantly greater than twice this estimated interaction distance. Even though the cyano groups do not physically touch, it is expected that the closer contact between them in the $\gamma$ phase would cause the frequency of modes to be higher in the $\gamma$ phase than in the $\beta$ phase. Results reported here show that the $\beta$-phase modes at 57 and $87 \mathrm{~cm}^{-1}$ shifted to the lower frequencies of 53 and 84 $\mathrm{cm}^{-1}$ in the $\gamma$ phase. This suggests that the cyano group interactions are not directly major contributors driving the $\beta-\gamma$ phase transition.

Another facet of the $\beta-\gamma$ phase transition is the dipoledipole interaction of malononitrile molecules in the lattice. At $25^{\circ} \mathrm{C}$, the molecular dipole moment, which is the bisector of the $\mathrm{C}-\mathrm{C}-\mathrm{C}$ angle in the plane of the cyano groups, is $3.56 \mathrm{D} .^{31}$ Its relatively high value is expected to influence the crystal packing and lattice vibrations. In the $\gamma$ phase, the angle between dipole moments for syn molecules is $39.92^{\circ}$, while in the $\beta$ phase, the angle is $37.99^{\circ}$. The angle between dipole moments for anti molecules are $140.08^{\circ}$ and $142.01^{\circ}$ for the $\gamma$ and $\beta$ phases, respectively. This suggests that the repulsive dipole interaction for syn molecules is increasing, while the repulsive dipole interaction is decreasing for anti molecules on going from the $\gamma$ to the $\beta$ phase. Calculation of the static dipole lattice sums shows the attractive interaction between anti molecules increases from -664 to $-736 \mathrm{~cm}^{-1} \AA^{-2}$ on transformation from the $\gamma$ to the $\beta$ phase, while the syn-molecule lattice sums of $-2360 \mathrm{~cm}^{-1} \AA^{-2}$ for the $\gamma$ phase and -2357 $\mathrm{cm}^{-1} \AA^{-2}$ for the $\beta$ phase show the dipole interaction stays essentially constant.

The similarities of malononitrile phase behavior to cyano based liquid crystal systems has been noted above. A ncmatic $\leftrightarrow$ smectic $A \leftrightarrow$ nematic re-entrant phase transition is observed in liquid crystals that display an antiparallel molecular association due to the large dipole moment associated with the cyano group. As the temperature is lowered from the nematic phase, the number of molecular associations increases, leading to the layered smectic $A$ 
phase. Something quite similar is seen in $\beta$-phase malononitrile where anti molecules oriented along the $c$ axis are stabilized by increased attractive dipole interactions. In the $\gamma$ phase, the increased repulsive dipole interactions between anti molecules lead to a less stable orientation along the $c$ axis, which is reminiscent of a nematic phase. Thus, the smectic $A \leftrightarrow$ nematic phase transition seen in cyanobased, liquid crystals and the $\beta-\gamma$ phase transition occurring in malononitrile may be driven by similar interactions between the molecular dipole moments associated with the cyano groups of the constituent molecules.

The dipole analyses indicate that the $\beta$ phase is more stable than the $\gamma$ phase. However, a lattice energy calculation by Rae and Dove shows that the $\gamma$ phase is more stable than the $\beta$ phase. ${ }^{32}$ This discrepancy can be rationalized by there being other processes besides cyano group and molecular dipole interactions involved in the phase transition. Even though this may be true, it is shown that these two processes can be used quite successfully to model the $\beta-\gamma$ phase transition.

Although the results presented here give no evidence for the existence of a $\hat{\mathbf{q}}=0$ optical soft mode, one may still exist. A previous $\mathrm{X}$-ray crystallographic study postulated a $b$-axis libration as the soft mode, ${ }^{3}$ while LeCalve et al. ${ }^{14}$ assigned an optical soft mode as a libration about the $C_{2}$ molecular axis of malononitrile. A librational mode about the unit cell $b$ axis, and thus about the molecular $C_{2}$ axis, is of $A_{g}$ symmetry. This study provides assignments of all six $A_{g}$-active Raman lattice modes in the $\gamma$ phase, none of which show soft mode behavior. Thus, any possible Raman active optical soft mode must have $B_{g}$ symmetry, which corresponds to librations about the $a$ or $c$ axes of the unit cell.

An x-ray analysis of the thermal vibrations of the malononitrile molecule shows that the rotation angle about the molecular $x$ axis, coincident with the unit cell $a$ axis, is much larger than are the rotations about the other two molecular axes. ${ }^{2}$ We postulate a possible $B_{g}$-active motion as the anti molecules librate in phase, while the syn molecules librate out of phase. This motion does not disturb the anti-molecule associations and allows large amplitude librations between molecules oriented along the $c$ axis. This $B_{g}$ symmetry libration corresponds to the thermal vibration analysis of the large amplitude libration ${ }^{2}$ and suggests that this would be the likely motion of any possible optical soft mode.

\section{v. CONCLUSION}

A temperature dependent, polarized Raman investigation from 4 to $200 \mathrm{~cm}^{-1}$ of the $\beta-\gamma$ phase transition occurring in sublimed, single crystal malononitrile is reported. In the pseudomonoclinic $\beta$ phase (triclinic) and monoclinic $\gamma$ phase, all six $A_{g}$-active modes, but only three of six possible $B_{g}$-active modes, were observed. In both phases, the assignment of the cyano-C-cyano bending mode of the malononitrile molecule at $181 \mathrm{~cm}^{-1}$ is made. Symmetry assignments of lattice vibrations in both phases were made solely from polarized, single-crystal Raman scattering results. The differences seen in mode frequencies from those of previous studies are attributed to differences in sample preparation and experimental methodologies. Spectra from sublimed, uncut, single crystals of malononitrile used in the above experiments are less likely to be affected by the degree of internal strain and defects inherent to melt-grown, cut crystals.

The temperature dependence of the lattice modes through the $\beta-\gamma$ transition showed that three modes are affected. For the $A_{g}$-active modes observed in the $\left(a c^{*}\right)$ configuration, the shifts are $57 \rightarrow 53 \mathrm{~cm}^{-1}$ and $87 \rightarrow 84$

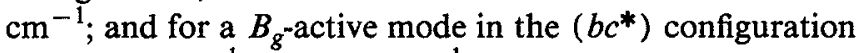
$111 \rightarrow 108 \mathrm{~cm}^{-1}$. The $57 \mathrm{~cm}^{-1}$ mode broadened with increasing temperature. This shifting and broadening is attributed to increased interaction between cyano groups on neighboring molecules and changes in the molecular dipole interactions. While a Raman-active, soft mode was not observed, the Raman data, along with crystal packing and dipole interaction analyses, suggest that a $B_{g}$-active librational motion about the unit cell $a$ axis could be an optical soft mode.

Malononitrile's $\beta-\gamma$ phase transition is shown to be reminiscent of the smectic $A \leftrightarrow$ nematic phase transition of certain cyano-based liquid crystal systems. The increased attraction between molecules in which the angle between molecular dipoles are greater than $90^{\circ}$ (anti molecules) leads to a $\beta$-phase structure which is more stable along the $c$ axis than in the $\gamma$ phase. This resembles the interactions of molecules in a liquid crystal smectic $A$ phase. The $\gamma$ phase exhibits greater dipole repulsion between anti molecules leading to less dipole stabilization along the $c$ axis. The $\gamma$ phase is thus analogous to a liquid crystal nematic phase. The interaction of the large molecular dipole of malononitrile leads to the response of the molecules through the phase transition. While the molecular dipole and cyano group interaction analyses model the $\beta-\gamma$ phase transition of malononitrile successfully, a comparison of experimental data to lattice energy calculations indicates these processes are not the only factors in determining the stability of both phases.

Although this study has clarified the nature of the $\beta-\gamma$ phase transition of malononitrile, there remain unresolved problems. Further investigation is certainly warranted, but must await approaches that will permit resolution of the high temperature phase spectra. The peculiar role of cyano groups in phase transitions in condensed phases also suggests further investigation.

\section{ACKNOWLEDGMENTS}

S.J.B. thanks the Center For Materials Research and Analysis of the University of Nebraska-Lincoln for financial support.

${ }^{1}$ A. Zussman and S. Alexander, J. Chem. Phys. 49, 3792 (1968).

${ }^{2}$ K. Obataki and S. Tanisaki, Phys. Lett. A 44, 341 (1973).

${ }^{3}$ M. T. Dove and A. I. M. Rae, Faraday Discuss. Chem. Soc. 69, 98 (1980).

${ }^{4}$ D. Bougeard, N. LeCalvé, and B. Pasquier, Ber. Bunsenges. Phys. Chem. 91, 1273 (1987).

${ }^{3}$ L. Tisza, Phase Transformations of Solids, edited by R. Smoluchowski, J. E. Meyer, and W. A. Weyl (Wiley, New York, 1951) Chap. 1. 
${ }^{6}$ P. E. Cladis, Phys. Rev. Lett. 35, 48 (1975).

${ }^{7}$ P. E. Cladis, D. Guillon, F. R. Bouchet, and P. L. Finn, Phys. Rev. A 23, 2594 (1981).

F. Hardouin, G. Sigaud, M. F. Achard, and H. Gasparoux, Phys. Lett. A 71,347 (1979).

${ }^{9} \mathrm{G}$. Sigaud, Nguycn Huu Tinh, F. Hardouin, and H. Gasparoux, Mol. Cryst. Liq. Cryst. 69, 81 (1981).

${ }^{10}$ H. L. Girdhar, E. F. Westrum, Jr., and C. A. Wulff, J. Chem. Eng. Data 13, 239 (1968).

"T. Fujiyama and T. Shimanouchi, Spectrochim. Acta 20, 829 (1964).

${ }^{12}$ E. Casteliucci and P. Manzelli, Chem. Phys. 9, 135 (1975).

${ }^{13}$ R. Savoie, R. Brousseau, and C. Nolin, Can. J. Chem. 54, 3293 (1976).

${ }^{14}$ N. LeCalvé, B. Pasquier, and A. Novak, J. Chem. Phys. 72, 6409 (1980).

${ }^{15}$ Y. Higashigaki and C. H. Wang, J. Chem. Phys. 71, 3813 (1979).

${ }^{16}$ N. Karl, in Crystals: Growth, Properties and Applications, edited by $\mathrm{H}$. C. Freyhardt (Springer, Berlin, 1980), Chap. 1.

${ }^{17} \mathrm{~F}$. Bloss, Introduction to the Methods of Optical Crystallography (Holt, Rinehart, and Winston, New York, 1961).

${ }^{18} \mathrm{~W}, \mathrm{~W}$. Wendlandt and H. G. Hecht, Reflectance Spectroscopy (Interscience, New York, 1966), Chap. 1.

${ }^{19}$ E. Castellucci, P. Manzelli, and R. Righini, Molecular Spectroscopy of
Dense Phases, edited by M. Grossman, S. G. Elkomoss, and J. Ringeissen (Elsevier, Amsterdam, 1976), p. 241.

${ }^{20}$ T. C. Damen, S. P. S. Porto, and S. B. Tell, Phys. Rev. 142, 570 (1966).

${ }^{21}$ M. Tobin, Laser Raman Spectroscopy (Wiley-Interscience, New York, 1971), Chap. 2.

${ }^{22}$ K. M. White, K.-H. Rrose, and C. I. Eckhardt, J. Chem. Phys. 85, 5551 (1986).

${ }^{23} \mathrm{R}$. Rudman, Low Temperature X-ray Diffraction (Plenum, New York, 1976), Chap. 3.

${ }^{24}$ T. Kawaguchi, A. Wakabayashi, M. Matsumoto, T. Takeuchi, and T. Watanabé, Bull. Chem. Soc. Jpn. 46, 57 (1973).

${ }^{25}$ T. Kawaguchi, K. Tanaka, T. Takeuchi, and T. Watanabé, Bull. Chem. Soc. Jpn. 46, 62 (1973).

${ }^{26}$ A. M. Golub, H. Köhler, and V. V. Skopenko, in Chemistry of Pseudohalides, edited by R. J. H. Clark (Elsevier, Amsterdam 1986), Chaps. 1 and 3.

${ }^{27}$ M. Ito, J. Chem. Phys. 42, 391 (1965).

${ }^{28}$ R. C. Dye and C. J. Eckhardt, J. Chem. Phys. 91, 3624 (1989).

${ }^{29}$ K. M. White and C. J. Eckhardt, J. Chem. Phys. 92, 2214 (1990).

${ }^{30} \mathrm{~L}$. Pauling, The Nature of the Chemical Bond, 3rd ed. (Cornell University, New York, 1960), p. 261.

${ }^{31}$ A. J. Fatiadi, Synthesis 3, 165 (1978).

${ }^{32}$ A. I. M. Rae and M. T. Dove, J. Phys. C 16, 3233 (1983). 